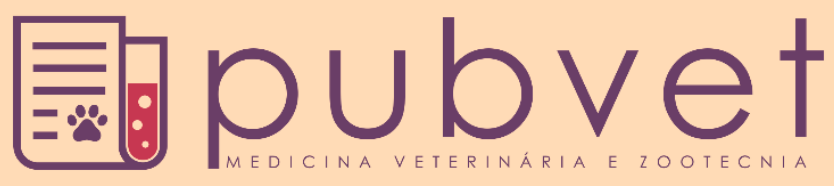

https://doi.org/10.31533/pubvet.v15n12a982.1-6

\title{
Gastropatia hipertrófica pilórica crônica: Revisão
}

\author{
Francesca Lopes Zibetti ${ }^{1} \bullet$, Eduardo Gonçalves da Silva ${ }^{1 *} \bullet$, Michaela Marques Rocha ${ }^{1} \bullet$, Caroline \\ Castagnara Alves $^{10}$, Brenda Madruga Rosa ${ }^{1} \bullet$, Daniele Weber Fernandes ${ }^{1} \bullet$, Maysa Seibert de \\ Leão $^{10}$, Matheus de Azevedo Soares ${ }^{10}$, Amanda Leal de Vasconcellos ${ }^{2}$, Paula Priscila Correia Costa $^{30}$ \\ ${ }^{I}$ Graduando de Medicina Veterinária, Universidade Federal de Pelotas, Pelotas-RS, Brasil. \\ ${ }^{2}$ Professor da Universidade Estadual do Ceará, Departamento de Clínicas Veterinárias, Fortaleza-CE, Brasil. \\ ${ }^{3}$ Professor da Universidade Federal de Pelotas, Departamento de Clínicas Veterinárias, Pelotas-RS, Brasil. \\ *Autor para correspondência, E-mail: goncalves-eduardo@outlook.com
}

Resumo. Existem duas formas de gastropatia hipertrófica pilórica crônica (GHPC), a de origem congênita, que é a hipertrofia muscular benigna do piloro, onde o aumento do volume celular ocorre nas células da camada muscular; e a adquirida, que consiste na hipertrofia da mucosa do antro gástrico, onde este aumento ocorre na camada mucosa; ambas provocam o retardo do esvaziamento gástrico por acarretar na estenose do lúmen pilórico. Este trabalho tem como objetivo trazer a revisão de literatura das formas de GHPC. O diagnóstico definitivo de ambas se dá por exames complementares de imagem, em especial a endoscopia e por biópsia; é muito importante se fazer o diagnóstico diferencial para demais enfermidades que apresentam sinais clínicos semelhantes, onde o principal deles é o vômito logo após a refeição. O tratamento de eleição é a correção cirúrgica pela técnica de piloroplastia, sendo necessário o acompanhamento do paciente no pós-cirúrgico e o prognóstico normalmente é favorável.

Palavras chave: Estenose, esvaziamento gástrico, exames de imagem, piloroplastia

\section{Chronic pyloric hypertrophic gastropathy: Review}

Abstract. There are two forms of chronic pyloric hypertrophic gastropathy (GHPC), the first one being of congenital origin, which is the benign pyloric muscle hypertrophy, where the increase in cell volume occurs in the cells of the muscle layer; and the second one being acquired, which consists of hypertrophy of the mucosa of the gastric antrum, where this increase occurs in the mucous layer; both cause delayed gastric emptying as it results in stenosis of the pyloric lumen. This work aims to bring the literature review of both forms of GHPC. The definitive diagnosis of both is made from complementary imaging exams, especially endoscopy and biopsy; it's very important to make the differential diagnosis for other diseases that present similar clinical signs, where the main one is vomiting right after the meal. The treatment of choice is surgical correction using the pyloroplasty technique, which requires monitoring the patient in the post-surgical period and the prognosis is usually favorable.

Keywords: Stenosis, gastric emptying, imaging exams, pyloroplasty

\section{Introdução}

A gastropatia hipertrófica pilórica crônica $(\mathrm{GHPC})$ é a forma genérica a qual se denomina à hipertrofia pilórica, sem que seja especificado se a camada muscular ou a mucosa fora acometida (Fossum, 2014), podendo ser uma patologia congênita ou adquirida. A hipertrofia da mucosa do antro gástrico é uma alteração adquirida que acomete mais frequentemente cães de pequenas raças, de meiaidade a idosos, e quando comparados com fêmeas, machos são mais acometidos (Cruz et al., 2016; 
Fossum, 2014; Walter \& Matthiesen, 1993). Já a alteração congênita, a hipertrofia muscular benigna do piloro, é mais comumente diagnosticada em animais jovens, sobretudo gatos siameses e cães braquicefálicos, entretanto pode ser encontrada em qualquer outra raça (Cruz et al., 2016). A GHPC diminui o tamanho do lúmen pilórico por provocar a estenose da região (Ettinger et al., 2002), sendo uma das possíveis causas em casos de retardo do esvaziamento gástrico (Cruz et al., 2016). A estenose pilórica, tanto por hipertrofia muscular quanto por hipertrofia da mucosa antral, é uma das alterações mais frequentes encontradas na região gástrica (Badi et al., 2004; Ceino-Gordillo et al., 2020).

Ainda a causa da GHPC é desconhecida, porém se tem como sugestão de possível desencadeador do processo a produção excessiva de gastrina. A gastrina é o hormônio principal responsável regular a secreção do ácido gástrico e tem taxia pela mucosa gástrica e pela musculatura lisa. Já se foi possível produzir em filhotes caninos a estenose pilórica congênita por administração de gastrina à gestante. Também tem um papel importante no estabelecimento da enfermidade a disfunção neurogênica, a qual pode causar a redução da motilidade gástrica e retenção do conteúdo digerido, as causas que podem causar este estímulo ao sistema nervoso simpático são diversas, como traumas, estresse agudo ou doenças inflamatórias. E igualmente, pode levar à hipertrofia a distensão gástrica prolongada por levar ao aumento de secreção de gastrina (Fossum, 2014).

O presente trabalho tem como objetivo fazer uma revisão de literatura a respeito de gastropatia hipertrófica pilórica crônica, salientando as diferenças das alterações congênitas e adquiridas, relacionando os achados de exames complementares e as possíveis causas de alterações.

\section{Histologia}

A parede do estômago é composta por quatro camadas: serosa ou adventícia externa, muscular externa, submucosa e mucosa interna (Köning \& Liebich, 2011). A camada serosa tem o revestimento denominado mesotélio, que consiste em epitélio pavimentoso simples e se constitui de uma camada fina de tecido conjuntivo frouxo. A muscular é formada por células musculares lisas dispostas em espiral, podendo ser desmembradas em duas subcamadas conforme a disposição das células; na mais externa a orientação se dá de forma longitudinal; já na mais interna, de forma circular (Junqueira \& Carneiro, 2013). A submucosa é constituída de tecido conjuntivo contendo vasos linfáticos e sanguíneos, além do plexo nervoso submucoso ou também chamado de plexo de Meissner; além disso pode conter tecido linfoide e/ou glândulas. A camada mucosa é a mais interna, que está em contato com o lúmen gástrico e tem variações conforme a região gástrica; de modo geral é composta por: revestimento epitelial, lâmina própria de tecido conjuntivo frouxo irrigado por vasos sanguíneos e linfáticos, contém células musculares lisas, podendo conter tecido linfoide e/ou glândulas; separando-a da submucosa existe a muscular da mucosa, a qual é constituída de duas subcamadas: uma longitudinal externa e uma circular interna; também é formada por epitélio glandular, se denomina fosseta gástrica a invaginação onde desembocam as unidades secretoras, as quais são tubulares e ramificadas, na região pilórica estas fossetas gástricas são profundas, onde pode conter glândulas tubulares simples ou ramificadas desembocando, também contém células $\mathrm{G}$ inseridas com as demais células da mucosa (Junqueira \& Carneiro, 2013).

Na GHPC ocorre a hipetrofia, ou seja, o aumento de volume das células da camada mucosa ou da muscular ou de ambas, na região pilórica; esta informação é essencial quando se for feita a biópsia do tecido aumentado de tamanho para a sua diferenciação de um processo neoplásico maligno (Fossum, 2014), que por definição consiste em um alto número de diferenciação de células parenquimatosas, podendo ser bem diferenciadas a completamente indiferenciadas, chamadas anaplásicas (umar, 2010).

\section{Anatomia}

O estômago está localizado entre o esôfago e o intestino delgado. Entre os mamíferos, apresenta grande variação de formato e distribuição dos diferentes tipos de mucosa. Quanto à forma podem ser divididos em unicavitário, onde há apenas um compartimento; ou pluricavitário, com diversos compartimentos (Köning \& Liebich, 2011).

Os cães e gatos possuem estômago unicavitário em forma de "C", a qual se é dividido em quatro regiões: parte cárdica, fundo gástrico, corpo gástrico e parte pilórica. Possui uma face visceral, a qual 
está voltada para a porção caudal e em contato com os órgãos abdominais contíguos, e outra parietal, posta intimamente contra fígado e diafragma; e possui também uma curvatura maior e outra menor (Köning \& Liebich, 2011). Sua localização é variável de acordo com seu grau de enchimento. O estômago está fixado na cavidade por cinco ligamentos: omento menor, omento maior, gastroesplênico, gastrofênico e gastropancreático (Oliveira, 2012).

A porção pilórica é pequena e está disposta em direção cranial e voltada dorsalmente; geralmente está situada oposta à porção ventral da nona costela ou espaço intercostal, tendo um alcance variável a direita do plano mediano, está relacionada com o pâncreas e com a fissura portal do fígado (Reece, 2008; Sisson et al., 1986).

\section{Hipertrofia muscular benigna do piloro}

Como citado anteriormente, a hipertrofia muscular benigna do piloro ou estenose pilórica hipertrófica congênita é mais comumente diagnosticada em animais jovens pelo fato de ser inato, porém pode ser observado em animais de todas as faixas etárias. Geralmente acomete cães braquicefálicos, como exemplo Buldogues, Boxers e Boston Terriers e gatos Siameses (Fossum, 2014). Causa alongamento e engrossamento do músculo pilórico, sendo trajada por uma hipertrofia concêntrica progressiva da musculatura do piloro juntamente com hiperplasia das células, sendo a sintomatologia clínica compatível com esta obstrução na saída do estômago (Lecompte et al., 2017). É uma das patologias gástricas de maior casuística (Ferreira et al., 2021; Thomson, 1990), sendo frequentemente diagnosticada através de gastroscopia (Cabrera \& Fernández, 2020; Tams \& Rawlings, 2010).

Comumente os animais acometidos vomitam a ingesta logo após a deglutição, descrito algumas vezes como "projétil". Podem-se apresentar clinicamente saudáveis, porém alguns podem demonstrar perda de peso. Determinados gatos com a estenose pilórica vomitam consideravelmente, podendo ser confundido seu quadro clínico com outras enfermidades, como regurgitação, esofagite secundária ou megaesôfago. Alguns casos podem ocorrer alcalose metabólica do tipo hipoclorêmica-hipocalêmica, todavia é incoerente e inespecífica para o diagnóstico de obstrução do fluxo gástrico, podendo ter outras causas, como consequência à terapia diurética agressiva (Nelson \& Couto, 2015). Na maioria das vezes os achados dos exames físicos são inespecíficos, podendo incluir anorexia, perda de peso, desidratação e/ou depressão, em contrapartida é rara a dor abdominal. Um quadro de pneumonia aspirativa e/ou refluxo esofágico, devido ao vômito crônico, podem ocorrer de forma secundária. A frequências dos vômitos é variável, de diversas vezes durante o dia à uma ou duas vezes semanais (Fossum, 2014). Geralmente os vômitos dos pacientes com hipertrofia muscular benigna do piloro começam quando estes passam a alimentar-se de sólidos, ou seja, logo após o desmame (Félix, 2011).

O diagnóstico é mais comum em cães ( clínico, todavia os exames complementares de imagem são indispensáveis para se fechar o diagnóstico (Penninck, 2004; Silva et al., 2013). O principal achado ultrassonográfico observado é o espessamento circunferencial do esfíncter pilórico, em especial da camada muscular (Penninck, 2004; Silva et al., 2013). As radiografias contrastadas podem expor o retardo do esvaziamento, defeito de preenchimento pilórico e/ou espessamento da parede do piloro. Porém, não se descarta obstrução quando há o esvaziamento natural do bário líquido, além disso, a mistura do bário ao alimento pode dificultar a interpretação da imagem. Ultimamente as radiografias contrastadas não são mais tão utilizadas para o diagnóstico de obstrução do fluxo gástrico, por conta da endoscopia além de ser tão sensível quanto, ainda permite ser feita a biópsia das lesões. Radiografias e ultrassonografias não tem precisão para diferenciar hipertrofia, inflamação ou neoplasia, já na endoscopia pode-se realizar a biópsia, onde geralmente se consegue se fazer esta distinção, sendo assim, um fator a evitar procedimentos cirúrgicos desnecessários (Fossum, 2014). As alterações bioquímicas e hematológicas no paciente com a afecção comumente são inespecíficas, caso o vômito ter acarretado em perda de secreções gástricas, pode estar presente hipocalêmia e alcalose metabólica hipoclorêmica; porém, é variável; pode ocorrer também, azotemia pré-renal; ou em cães jovens em estado mais grave, pode ser visto hipoalbunemia leve (Fossum, 2014).

Como diagnóstico diferencial, pode-se ter qualquer situação que cause vômito, como por exemplo, neoplasia, inflamação, ulceração, pitiose e corpos estranhos gastrointestinais podem levar à obstrução do fluxo gástrico. Outras possíveis causas devem ser eliminadas antes de se realizar um procedimento 
cirúrgico: hipoadrenocorticismo, uremia, hipercalemia, peritonite, hipertireoidismo felino, cetoacidose diabética, pancreatite, insuficiência hepática, gastrite, doença inflamatória da bexiga e falência cardíaca precoce do lado direito em felinos (Fossum, 2014).

O tratamento mais indicado é a correção cirúrgica através da piloroplastia, como exemplo de técnica a se utilizar tem-se a plastia Y-U, que tem mais efetividade do que a piloromiotomia. Todavia, ambos procedimentos mal realizados podem causar obstrução ou perfuração gástrica (Nelson \& Couto, 2015). O objetivo da técnica é remover a obstrução para que reestabeleça o esvaziamento gástrico habitual. Deve ser encaminhado material da espessura completa para biópsia para certificar-se da benignidade do espessamento. Mesmo com caráter benigno, pólipos que estiverem sangrando ou obstruindo o fluxo gástrico devem ser removidos (Fossum, 2014). A técnica de piloroplastia em Y-U consiste em uma incisão tendo formato de $\mathrm{Y}$ que inclua todas as camadas celulares do piloro, onde as duas linhas estejam paralelas às curvaturas menor e maior do estômago e a sutura em forma de $\mathrm{U}$, sendo utilizado ponto simples com fio absorvível do tipo monosyn 3/0 redondo (Félix, 2011; Fossum, 2014). Nesta técnica cirúrgica uma parte da parede antral é transposta a região pilórica, com a aplicação desta se faz a ampliação do lúmen pilórico (Cruz et al., 2016). Antes da realização dos procedimentos cirúrgicos ou endoscópicos, as anormalidades eletrolíticas, desequilíbrio ácido-básico e desidratação devem ser corrigidas. $\mathrm{O}$ uso de fármacos inibidores da bomba de prótons podem ser realizados em pacientes com esofagite decorrente a exposição frequente da mucosa esofágica ao suco gástrico. Para aspiração ou ulceração pode ser indicado o uso de antibióticos. A administração de pró-cinéticos gástricos, como cisaprida ou metoclopramida, não devem ser cogitadas em suspeita clínica de obstrução de fluxo gástrico (Fossum, 2014). Geralmente o prognóstico é bom, porém determinados pacientes no pós-cirúrgico carecem de refeições com pouca quantidade e maior frequência, com o suporte de fármacos prócinéticos, como os já citados anteriormente. Quando não há causa metabólica ou mecânica no retardo funcional, o tratamento tem como fundamento uso de alimentação específica e utilização de prócinéticos. O fármaco com maior eficácia é a cisaprida, é um pró-cinético que coordena as contrações tanto do antro gástrico, como piloro e duodeno, faz com que aumente o tempo entre contrações duodenais, não causando efeitos adversos. Também acelerando o esvaziamento gástrico se tem a metoclopramida, a qual pode ser administrada em casos de alterações de motilidade onde a causa seja metabólica, podendo estimular o centro do vômito. Ranitidina e nizatidina são antagonistas de receptores H2 que podem ser utilizados como pró-cinéticos, tendo efeitos de estimular tanto esvaziamento gástrico quanto motilidade do intestino delgado e do intestino grosso, além de inibir a secreção do ácido no estômago; são indicadas em casos de diminuição de motilidade secundária à inflamação ou ulceração. Baixas doses de eritromicina diminuem as contrações antrais e acelera o esvaziamento gástrico (Jericó et al., 2015).

\section{Hipertrofia da mucosa do antro gástrico}

Como relatado anteriormente, a hipertrofia da mucosa antro gástrico ou hipertrofia da mucosa pilórica é uma afecção adquirida, acomete principalmente raças de pequeno porte, ou seja, com menos de $10 \mathrm{~kg}$, em especial Malteses, Lhasa Apso e Shih Tzu, sendo machos mais acometidos em relação as fêmeas, e animais em meia-idade os mais susceptíveis, podendo disfarçar uma neoplasia. Determinados caninos já relatados com a afecção foram avaliados com comportamentos viciosos ou muito excitáveis (Fossum, 2014). Em aspecto clínico, a hipertrofia da mucosa antral e a estenose pilórica assemelhamse, os pacientes vomitam o alimento após as refeições (Nelson \& Couto, 2015).

O diagnóstico se dá por radiografia, ultrassonografia ou endoscopia; porém para o diagnóstico definitivo é necessário a realização de biópsia. Apenas por endoscopia, a hipertrofia na mucosa pode ser confundida com neoplasia de submucosa por suas semelhanças em causar dobras convolutas na mucosa. Alguns pacientes estão com a mucosa nitidamente inflamada e avermelhada, porém não é rígido ou firme como o acreditado aos animais com leiomiomas ou carcinomas infiltrativos. Porventura a hipertrofia da mucosa antral só seja visualizada durante um procedimento cirúrgico, deve-se tentar diferenciar de neoplasia por infiltração na submucosa ou de hipertrofia benigna do piloro por espessamento muscular. Sendo de extrema importância ser feito o diagnóstico diferencial para outras possíveis causas para a escolha da terapêutica apropriada (Nelson \& Couto, 2015). Também, como 
diagnóstico diferencial, se mantém os mesmos que para hipertrofia muscular benigna do piloro, assim como os achados em radiografias contrastadas com bário e alterações hematológicas (Fossum, 2014).

O tratamento é a ressecção da mucosa, normalmente associada a piloroplastia, já a técnica isolada de piloromiotomia se torna na maioria dos casos ineficiente para a resolução dos sinais clínicos da patologia (Nelson \& Couto, 2015), e o prognóstico para a hipertrofia da mucosa do piloro é bom (Fossum, 2014).

\section{Considerações finais}

Como em todas as afecções, o diagnóstico definitivo preciso é de grande importância, diferenciar das demais possíveis causas e descartá-las através da análise clínica, histórico do paciente e principalmente exames complementares de imagem e biópsia, são fundamentais para se fechar diagnóstico de hipertrofia muscular benigna do piloro ou hipertrofia da mucosa do antro gástrico. Ambas patologias têm um prognóstico muito favorável após o tratamento com a correção cirúrgica do espessamento do piloro pela técnica de piloroplastia, além de acompanhar a evolução do paciente no pós-cirúrgico. Sempre se faz importante a competência profissional para um diagnóstico clínico preciso e rápido, melhor escolha de tratamento para que se obtenha êxito e proporcione conforto ao paciente.

\section{Referências bibliográficas}

Badi, H. N., Yazdani, D., Ali, S. M., \& Nazari, F. (2004). Effects of spacing and harvesting time on herbage yield and quality/quantity of oil in thyme, Thymus vulgaris L. Industrial Crops and Products, 19(3), 231-236. https://doi.org/10.1016/j.indcrop.2003.10.005

Cabrera, J. S., \& Fernández, S. A. (2020). Uso de la endoscopia en medicina interna de pequeños animales. Basado en la evidencia científica. Revista Centro Universitário, 92, 6-21.

Ceino-Gordillo, F., Ortiz-Huaranga, X., Castro-Moreno, D., Pineda, R., \& Tolentino-Camarena, M. (2020). Afecciones más frecuentes en caninos en clínicas veterinarias de Lima, Perú. Biotempo, 17(2), 227-235. https://doi.org/10.31381/biotempo.v17i2.3308.

Cruz, T. P. P. S., Ruiz, T., Campos, W. N. S., Azevedo, L. S., Bicudo, G. A., Néspoli, P. B., \& Souza, R. L. (2016). Piloroplastia em YU para a correção da gastropatia hipertrófica pilórica crônica em caninos. Acta Scientiae Veterinariae, 44, 1-5. https://doi.org/10.22456/1679-9216.83122.

Ettinger, S. J., Fedlman, E. C., \& Taibo, R. A. (2002). Tratado de medicina interna veterinaria: enfermedades del perro y el gato. Manole.

Félix, A. (2011). Estenose pilórica congénita num bulldog francês-caso clínico. Revista Lusófona de Ciência e Medicina Veterinária, 4, 23-27. https://doi.org//10437/2267.

Ferreira, D. F., Fernandes, C. C., \& Dias, A. S. (2021). Levantamento das alterações do trato gastrointestinal em cães submetidos a necropsia na Universidade Presidente Antônio Carlos, em Uberlândia-MG. Scientific Electronic Archives, 14(3), 94-98. https://doi.org/10.36560/14320211238.

Fossum, T. W. (2014). Cirurgia de pequenos animais (4th ed., Vol. 1). Elsevier Brasil.

Hall, J. A., Willer, R. L., Seim, H. B., \& Lebel, J. L. (1992). Gastric emptying of nondigestible radiopaque markers after circumcostal gastropexy in clinically normal dogs. American Journal Veternary Research, 53(10), 19-61.

Jericó, M. M., Kogika, M. M., \& Andrade Neto, J. P. (2015). Tratado de medicina interna de cães e gatos. Guanabara Koogan.

Junqueira, L. C., \& Carneiro, J. C. (2013). Histologia Básica (12 ed.). Guanabara Koogan.

Köning, H. E., \& Liebich, H. G. (2011). Anatomia dos animais domésticos texto e atlas colorido. Editora Artmed.

Kumar, V. (2010). Robbins \& cotran-patologia bases patológicas das doenças. Elsevier Brasil.

Lecompte, J.-F., Gastaldi, P., \& Bréaud, J. (2017). Estenosis hipertrófica del píloro. EMC-Pediatría, 52(3), 1-6. https://doi.org/10.1016/S1245-1789(17)85525-6.

Nelson, R. W., \& Couto, C. G. (2015). Medicina interna de pequenos animais (Issue 1). Elsevier Editora.

Oliveira, A. L. A. (2012). Técnicas cirúrgicas em pequenos animais. Elsevier Brasil. 
Penninck, D. G. (2004). Trato gastrointestinal. In T. . Nyland \& J. S. Matton (Eds.), Ultrassom diagnóstico em pequenos animais (pp. 211-234). Roca, Brasil.

Reece, W. O. (2008). Anatomia funcional e fisiologia dos animais domésticos. Editora Roca.

Silva, L. C., Belotta, A. F., Machado, V. M. V., \& Vulcano, L. C. (2013). Avaliação ultrassonográfica gástrica em pequenos animais. Veterinária e Zootecnia, 20, 567-575.

Sisson, S., Grossman, J. D., \& Getty, R. (1986). Anatomia dos animais domésticos. Interamericana.

Tams, T. R., \& Rawlings, C. A. (2010). Small animal endoscopy-E-Book. Elsevier Health Sciences.

Thomson, R. G. (1990). Patologia veterinária especial (Vol. 1). Manole.

Walter, M. C., \& Matthiesen, D. T. (1993). Acquired antral pyloric hypertrophy in the dog. Veterinary Clinics of North America: Small Animal Practice, 23(3), 547-554. https://doi.org/10.1016/S01955616(93)50304-2.

Histórico do artigo:

Recebido: 6 de agosto de 2021

Aprovado: 5 de setembro de 2021

Disponível online: 9 de novembro de 2021
Licenciamento: Este artigo é publicado na modalidade Acesso Aberto sob a licença Creative Commons Atribuição 4.0 (CC-BY 4.0), a qual permite uso irrestrito, distribuição, reprodução em qualquer meio, desde que o autor e a fonte sejam devidamente creditados. 